\section{Systemic chemotherapy with oxaliplatin is a good option for advanced hepatocellular carcinoma}
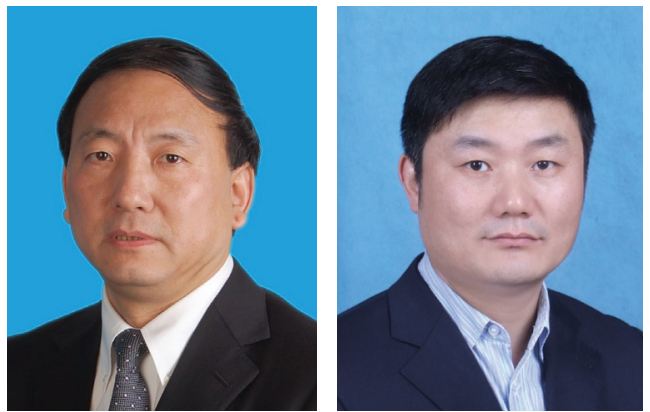

\author{
"Systemic therapy for \\ hepatocellular carcinoma is \\ an evolving field in past years \\ and appropriate for patients \\ with advanced unresectable \\ disease who are unsuitable \\ for locoregional therapy."
}

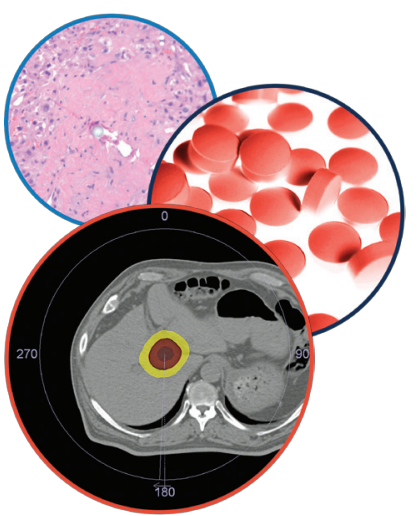

Hepatic Oncology

Shukui Qin ${ }^{*}$ \& Qiong Wu²

Hepatocellular carcinoma (HCC) is the fifth most common solid malignancy and the third leading cause of cancer death worldwide, with about 800,000 new cases diagnosed per year globally, in which $83 \%$ in the less developed countries or regions (more than 50\% in China alone) [1-3].

In Asia, especially China, HCC is one of the most common cancers, the incidence is still high and rising, mainly due to hepatitis B virus infection, water pollution by microcystin in rural areas, alcohol abuse (excessive drinking) and food pollution by aflatoxin [2]. It is well known that HCC between Asian and western countries is obviously different with respect not only to their etiology and epidemiology, but also to their biologic behavior, clinical staging, manifestation, treatment strategy, clinical practice guidelines and prognosis etc. Thus, HCC between Asian and western countries is very heterogeneous and often considered as 'two kind of diseases.'

HCC is always deemed as one of the most aggressive tumors that frequently occurs in the setting of chronic liver disease and cirrhosis, progressing silently, rarely producing obvious symptoms until the advanced stage. Most HCC patients $(\sim 80 \%)$ especially in Asia region including China, often present with advanced disease (BCLC stage C and D) and lose the chance of curative hepatectomy or transhepatic arterial chemptherapy and embolization (TACE), and other localized treatment at diagnosis. Those advanced HCC patients thus have a very poor prognosis and median survival with supportive care alone of approximately only 3-4 months in China and Eastern Asian countries, but 6-9 months in western countries.

Systemic therapy for HCC is an evolving field in past years and appropriate for patients with advanced unresectable disease who are unsuitable for locoregional therapy. Sorafenib is a multikinase inhibitor acting on HCC via both antiproliferative and antiangiogenic effects. Sorafenib is the first systemic anticancer drug to demonstrate a survival benefit compared with

\section{KEYWORDS}

- hepatocellular carcinoma

- oxaliplatin • systemic

chemotherapy $\bullet$ treatment

'Chinese PLA Cancer Center, Nanjing Bayi Hospital, Nanjing 210002, China

${ }^{2}$ The Medical Department, The First Affiliated Hospital of Bengbu Medical College, Bengbu 233004, China

*Author for correspondence: Tel.: +8625 8445 3932; qinsk@csco.org.cn

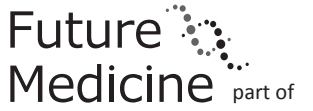

“For advanced hepatocellular carcinoma patients ... systemic chemotherapy is commonly used as a palliative method in clinical practice of Asian and some western counties...” 
“...oxaliplatin-based chemotherapy is effective in advanced hepatocellular carcinoma and represents a viable option in these patients.” best supportive care alone in two international Phase III studies (SHARP and ORIENTAL trials) which represented a great milestone in HCC treatment $[3,4]$. After that break through treatment, molecular-targeted therapy becomes the frontier and hot issue in the clinical practice and research. A series of new molecularly targeted drugs, including sunitinib bravinib, lapatinib, linifanib, everolimus and axitinib etc., were studied one by one, but unfortunately all failed in their Phase III trial. Even the trials of sorafenib plus erlotinib and sorafenib combining with TACE also got negative results. This maybe because of that HCC has the complexity of etiology, process and mechanism, particularly the background liver disease and especially lack the experience of clinical research and the lesson not learned. Up to date, Sorafenib is still the only one supported by strong evidence of Phase III clinical study in HCC. Again sorafenib has several big disadvantages, restricting its wide application, especially in China. Its disadvantages include very low response rate (RR) of only $2-3 \%$, the life-prolonging effect limited to a few months, for example, a very limited survival benefit, no difference in time to tumor symptom progression, for example, without significantly improving of main symptoms, a wide range of toxicities, such as hand-foot reaction, bleeding, hypertension, diarrhea and skin rash, very expensive (50,000 Chinese Yuan RMB per month in China) and lack of the molecular biomarker to predict drug efficacy or toxicities to choose the right patients. Thus a great unmet need remains for HCC patients who are ineligible for, intolerant to, or progress during sorafenib treatment.

For advanced HCC patients who are ineligible for surgery or localized treatments, systemic chemotherapy is commonly used as a palliative method in clinical practice of Asian and some western counties for many years, in order to reduce related symptoms improve quality of life, control tumor spread or shrink tumor and prolong overall survival. In the past, cisplatin (PDD), 5-fluorouracil and doxorubicin (DOX) are the most commonly used, but without both standard drugs or regimen and high level evidence of survival benefit. A lot of Phase II and Phase III systemic chemotherapy studies all failed. Because the majority of HCC patients have basic liver disease (hepatitis and liver cirrhosis). Their liver function has been damaged, limiting the drug dose intensity and/or density and affecting their effect. Traditional chemotherapy drugs, including PDD, 5-fluorouracil and DOX, because of itself obvious toxicities, offset or cover up its benefit. Most previous systemic chemotherapy trials have not stratified patients according to some very important prognostic factors, such as countries or regions, HBV or HCV infection, extrahepatic lesions and/or vascular invasion, liver dysfunction (ChildPugh score), liver fibrosis or cirrhosis, BCLC stage and ECOG status, etc. Thus data were negatively impacted by heterogeneous HCC populations, co-morbidities and background liver disease. It was considered that advanced HCC is highly resistant to those old cytotoxic chemotherapy drugs.

In recent years, some new generation chemotherapy drugs with high efficacy and low toxicities, such as oxaliplatin (OXA), capecitabine and gemcitabine, are widely and successfully used in clinical, making great progression, improving significantly the prognosis in gastrointestinal cancers and pancreatic cancer. Those inspired and promoted the research of systemic chemotherapy for HCC, bringing out the new situation. So, the traditional and old concept of chemotherapy unsuitable for HCC has meet strong doubts and great challenges.

OXA is a third-generation platinum agent with obvious advantages. OXA-containing regimens have shown improved clinical activity against HCC in several Phase II studies and clinical real world [2]. The data of a number of Phase II clinical trial for patients with advanced HCC (total number of cases 2700 ) suggested that OXA-containing regimens are effective with RR 3-27\%, disease control rate (DCR) $42-89 \%$, median time to progression (mTTP) 2-8 months and median overall survival ( $\mathrm{mOS}$ ) 4-12 months with better safety and tolerance.

In an international, multicenter, open-label, randomized, Phase III trial (EACH study), compared with DOX alone, oxaliplatin plus 5-FU/leucovorin (FOLFOX4) regimen significantly improved PFS, RR and DCR, and had the improvement tendency of overall survival (OS) in Asian advanced HCC, especially for Chinese patients $[5,6]$. In the EACH study 371 patients with advanced HCC from 38 sites in the Asian-Pacific region have been enrolled. Of the patients enrolled, $75 \%$ were from China mainland and Taiwan area, 14\% from Korea and $11 \%$ from Thailand. Patients were randomly assigned to receive either FOLFOX4 $(n=184)$ 
or DOX $(\mathrm{n}=187)$ until disease progression, intolerable toxicity, death, patient withdrawal of consent or until the patient became eligible for surgical resection, whichever occurred first. The FOLFOX4 regimen significantly improved the median progression-free survival (mPFS) 1.77 months in DOX group versus 2.93 months in FOLFOX4 group ( $<<0.001$ ), RR: $2.67 \%$ in DOX group versus $8.15 \%$ in FOLFOX4 group $(\mathrm{p}=0.02)$ and DCR: $31.55 \%$ in DOX group versus $52.17 \%$ in FOLFOX4 group $(\mathrm{p}<0.001)$ when compared with DOX alone. The mOS at the first analysis time point was also longer in the FOLFOX4 group although it did not reach statistical significance (4.97 months in DOX group vs 6.40 months in FOLFOX4 group; $\mathrm{p}=0.06$ ). After an additional 7 months of follow-up, the benefit on mOS become significant favoring the FOLFOX4 group (6.47 months in FOLFOX4 group vs 4.90 months in DOX group, $\mathrm{p}=0.04)$. For the Chinese patients population, the median OS was significantly longer in the FOLFOX4 group than in DOX alone group at the both cut-off dates. The median OS at the first prespecified time point of treatment was 5.7 months with FOLFOX 4 and 4.3 months with DOX group $(\mathrm{p}=0.03)$. At the end of the follow-up period, the mOS was 5.9 months with FOLFOX4 and 4.3 months with DOX group ( $\mathrm{p}=0.03$ ). Additionally, the mPFS, the RR and the DCR were significantly higher in the FOLFOX4 group than in the DOX group (mPFS: 2.4 vs 1.7 months, $\mathrm{p}=0.0002$; RR: 8.6 vs $1.4 \%, p=0.006$; DCR: 47.1 vs $26.6 \%$, $\mathrm{p}=0.0004)$. In terms of toxicity, the incidence of neutropenia and neurotoxicity was higher in the FOLFOX 4 group, but the incidence of grade $3 / 4$ toxicities was similar in both treatment groups. Overall, the reported adverse events were consistent with those reported in previous clinical trials of FOLFOX4 in patients with colorectal cancer with no unexpected adverse events. As compared with results reported with sorafenib in the ORIENTAL trial [4] which also enrolled HCC patients from the Asia-Pacific region, the results of the whole population or Chinese subgroup analysis of the EACH study were of similar magnitude in terms of PFS and OS, but with better results in terms of RR and DCR $[5,6]$.

The results of the EACH study were supported by the results of several other studies performed in Chinese patients [7-9]. Such as in a single-center study later, the FOLFOX4 regimen was administered as systemic chemotherapy in 77 Chinese patients with advanced HCC with or without previous chemotherapy for HCC [7]. The RR and DCR were 4.2 and $55.6 \%$, respectively. mTTP was 2.7 months and mOS was 6.1 months. The stratified analysis showed a similar effect of this regimen both in untreated patients and in the patients who previously received chemotherapy, but with a lower efficacy in the patients with portal vein invasion or extra hepatic metastasis. The common adverse events were also leucopenia and mild peripheral neurotoxicity.

Based on the current clinical practice in China and the urgent medical demand of HCC, the findings from EACH study, along with other evidences supporting OXA use, have led to systemic chemotherapy with FOLFOX4 being included in Chinese national clinical practice guidelines for HCC [10] and got Chinese FDA approval for advanced HCC indication in China in 2013 [11]. Therefore, the basic research and clinical study of systemic chemotherapy with OXA has become a hotspot in the field of HCC treatment.

In some western countries, OXA-containing regimens were also studied. Such as AGEO study is other large, multicenter, retrospective study to observe GEMOX regimen for advanced HCC in France, with total of 204 patients between 2001 and 2010 [12]. Its results also shown RR 22 as well as DCR 66\%, mPFS, mTTP and mOS were 4.5 (95\% CI: 4-6), 8 (95\% CI: 6-11) and 11 months (95\% CI: 9-14), respectively, and $8.5 \%$ of the patients were subsequently eligible for curative-intent therapies after down staging. GEMOX regimen may improve RR, DCR, PFS, TTP and OS. Multiplefactor analysis shown that gender $(\mathrm{p}=0.03)$, background cirrhosis $(\mathrm{p}=0.01)$, CLIP score $(\mathrm{p}=0.03)$ and efficiency of GEMOX ( $<<0.0001)$ are independent factors associated with OS. Toxicities shown $44 \%$ patients had grade 3/4 toxicities, in which thrombocytopenia occupied $24 \%$, granulopenia $18 \%$, diarrhea $14 \%$, neuropathy $12 \%$ and leading to treatment discontinuation in $16 \%$ of the cases. Still there were some observations shown that for HCC patients resistant to sorafenib with progression of disease, changing to or combining systemic chemotherapy with OXA-containing regimens may play some beneficial role again. Recent experimental studies have shown that OXA has new mechanism against cancer cell with non-DNA-binding effects, that is, inducing immunogenic cell death [13,14].
"New, better drugs and therapeutic strategy including systemic chemotherapy combining with molecular target therapy drugs should be highly considered and intensively investigated in the near future." 
Petrelli et al. have published a systemic review and pooled analysis named 'OXA-based chemotherapy: a new option in advanced hepatocellular carcinoma.' In the paper normative 13 studies were collected, with a total of 800 patients analyzed [15]. The RR was about $16.8 \%$. The mPFS and mOS were 4.2 and 9.3 months, respectively, with a one-year overall survival of $37 \%$. The weighted $\mathrm{mPFS} / \mathrm{mOS}$ and $\mathrm{RR}$ were $4.5 / 11$ months and $20 \%$ respectively in western patients. Conversely, in Asiatic studies, the $\mathrm{mPFS} / \mathrm{mOS}$ and RR were 2.43/6.47 months and $13.2 \%$ respectively in Asian patients. Thus, the authors pointed out that 'OXA-based chemotherapy is effective in advanced HCC and represents a viable option in these patients. A head to head comparison with sorafenib or a second-line agent should be verified in prospective trials'.

Therefore, new systemic OXA-combination chemotherapy with better response is another option for some advanced HCC patients with distal metastasis or patients who are not candidates for local/regional treatment. And for those patients unsuitable for sorafenib or after sorafenib failure, systemic chemotherapy maybe a useful alternative treatment and deserve further investigations.

In Chinese national clinical practice guidelines for HCC, there are strict indication and contraindication for using systemic chemotherapy. The indication of systemic chemotherapy for HCC include: those patients who were already advanced with extra hepatic metastases; local lesion, but not suitable for surgical resection, radiofrequency ablationor microwave ablation, and TACE, or progress after above localized treatment; diffuse type HCC; complicated by tumor emboli in portal venous and/or inferior vena cava. The contraindication include: ECOG $>2$, Child-Pugh core $>7$; WBC $<3.0 \times 10^{9} / 1$ or ANC $<1.5 \times 10^{9} / 1, \mathrm{BPC}<60 \times$ $10 \% / \mathrm{LHb}<90 \mathrm{~g} / \mathrm{l}$; liver and/or renal function dysplasia, AST or ALT $>5 \mathrm{ULN}$ and/or bilirubin $>2$ ULN, serum albumin $<28 \mathrm{~g} / \mathrm{l}, \mathrm{Cr} \geq 1$
ULN, CCr $\geq 50 \mathrm{ml} / \mathrm{min}$; the patient has the active infection, fever, bleeding tendency, in a large number of peritoneal effusion and hepatic encephalopathy [10]. Besides those above conditions, it should be emphasized that when systemic chemotherapy is used for advanced HCC patient, we should pay much more attention to monitor HBV and/or HCV activity and anti$\mathrm{HBV} / \mathrm{HCV}$ drug must be used if $\mathrm{HBV}$ and/or $\mathrm{HCV}$ are active, and to protect liver function rightly [16].

In conclusion, HCC remains a highly lethal disease, continues to be a major healthcare burden in whole world with huge potential patient population, and extremely endemic in Asia including China. Treatment options are limited and medical needs remain very high. The traditional and old concept of chemotherapy unsuitable for HCC has met strong doubts, great challenges and changes. Now, new systemic combination chemotherapy with better response is another option for some advanced HCC patients with distal metastasis or patients who are not candidates for local/regional treatment [17]. Of course, systemic chemotherapy is only a means in the comprehensive treatment of advanced HCC, may only solve part of the problems, its effect is also limited. There is still a long way to go. New, better drugs and therapeutic strategies including systemic chemotherapy combined with molecular target therapy drugs should be highly considered and intensively investigated in the near future [18-20]. We should try our best to promote it to conquer HCC.

Financial \& competing interests disclosure The authors have no relevant affiliations or financial involvement with any organization or entity with a financial conflict with subject matter or material discussed in the manuscript. This includes employment, consultancies, honoraria, stock ownership or options, expert testimony, grants or patents received or pending, or royalties.

No writing assistance was utilized in the production of this manuscript.

\section{References}

1 Torre LA, Bray F, Siegel RL et al. Global cancer statistics 2012. CA Cancer J. Clin. 65(2), 87-108 (2015).

2 Gong XL, Qin SK. Progression of clinical and laboratory studies on systemic chemotherapy with oxiliplatin-combined regimens in advanced hepatocellular carcinoma. Chin. J. Front. Med. Sci. 6, 22-33 (2014).
3 Llovet JM, Ricci S, Mazzaferro V et al. Sorafenib in advanced hepatocellular carcinoma. N. Engl. J. Med. 359, 378-390 (2008).

4 Cheng AL, Kang YK, Chen Z et al. Efficacy and safety of sorafenib in patients in the Asia-Pacific region with advanced hepatocellular carcinoma: a Phase III randomised, double-blind, placebo-controlled trial. Lancet Oncol. 10, 25-34 (2009).
5 Qin SK, Bai YX, Lim HY et al. Randomized, multicenter, open-label study of oxaliplatin plus fluorouracil/leucovorin versus doxorubicin as palliative chemotherapy in patients with advanced hepatocellular carcinoma from Asia. J. Clin. Oncol. 31, 3501-3508 (2013).

6 Qin SK, Cheng Y, Liang J et al. Efficacy and safety of the FOLFOX4 regimen compared with doxorubicin in Chinese patients with 
advanced hepatocellular carcinoma: a subgroup analysis of the EACH study. Oncologist 19, 1169-1178 (2014).

7 Yang LQ, Qin SK, Zhao NL et al. Clinical study on FOLFOX 4 regimen as systemic chemotherapy for advanced primary liver carcinoma. Chin. Clin. Oncol. 18, 108-113 (2013).

8 Zhu H, Ping Sun. Clinical study of FOLFOX4 regimen for patients of advanced hepatocellular carcinoma. Chinese-German J. Clin. Oncol. 11, 134-137 (2012).

9 Chen G, Chen H, Qin L et al. Intra-tumoral injection of E1B gene-deleted adenovirus combined with FOLFOX4 for advanced hepatocellular carcinoma. J. Diagn. Imag. Interv. Radiol. 21, 137-140 (2012).

Chinese Ministry of Health. Diagnosis and Treatment Guideline of Primary Liver Cancer (Ed 2011). Chinese Ministry of Health, Beijing, China (2011). http://d.wanfangdata.com.cn/Periodical_ lcgdbzz201111004.aspx
11 Oxaliplatin CFDA approval for treatment of advanced HCC. http://drug.39.net/a/130412/4157014.html

12 Zaanan A, Williet N, Hebbar M et al. Gemcitabine plus oxaliplatin in advanced hepatocellular carcinoma: a large multicenter AGEO study. J. Hepatol. 58, 81-88 (2013).

13 Alcindor T, Beauger N. Oxaliplatin: a review in the era of molecularly targeted therapy. Curr. Oncol. 18, 18-25 (2011).

14 Ramakrishnan R, Assudani D, Nagaraj S et al. Immunogenic death of colon cancer cell treated with oxaliplatin. Oncogen 29, 482-491 (2010).

15 Petrelli F, Coinu A, Cabiddu M et al. Oxaliplatin-based chemotherapy: a new option in advanced hepatocellular carcinoma. A systematic review and pooled analysis. Clin. Oncol. 26, 488-496 (2014). Ye SL, Jiang JJ, Dong Q et al. Antiviral treatment in HBV/HCV related HCC expert consensus. Chin. J. Liver Dis. 22, 321-325 (2014).
17 Chan SL. Drug development for hepatocellular carcinoma: knowing the past help to understand the future. Oncolgist 19, 1-3 (2014).

18 Assenat $\mathrm{E}$, Boige $\mathrm{V}$, Thézenas $\mathrm{S}$ et al. Sorafenib (S) alone versus $S$ combined with gemcitabine and oxaliplatin (GEMOX) in first-line treatment of advanced hepatocellular carcinoma (HCC): final analysis of the randomized Phase II GONEXT trial (UNICANCER/FFCD PRODIGE 10 trial). J. Clin. Oncol. 31(Suppl.), Abstract 4028 (2013).

19 Yau T, Cheung F, Lee F et al. A multicenter Phase II study of sorafenib, capecitabine, and oxaliplatin (SECOX) in patients with advanced hepatocellular carcinoma: final results of Hong Kong-Singapore Hepatocellular Carcinoma Research Collaborative Group study. J. Clin. Oncol. 31(Suppl.), Abstract 4117 (2013).

20 Harding JJ, Abou-Alfa GK. Systemic therapy for hepatocellular carcinoma. Chin. Clin. Oncol. 2(4), 37-44 (2013). 